\title{
Carga de trabalho de enfermagem em uma unidade de terapia intensiva de trauma*
}

\author{
Nursing workload in a trauma intensive care unit
}

Carga de trabajo de enfermería en una unidad de terapia intensiva de trauma

Luana Loppi Goulart ${ }^{1}$, Roberta Nazário Aoki ${ }^{2}$, Camila Fernanda Lourençon Vegian ${ }^{3}$, Edinêis Brito Guirardello ${ }^{4}$

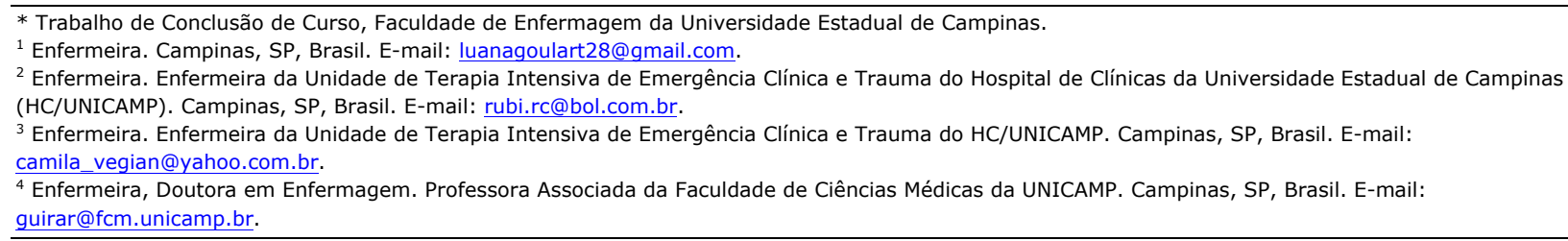

\section{RESUMO}

Pacientes gravemente feridos, com lesões múltiplas e conflitantes, apresentam-se aos enfermeiros de unidades de cuidados críticos como desafios na gestão da assistência. O objetivo deste estudo foi avaliar a carga de trabalho de enfermagem e verificar a correlação entre a carga e o índice de gravidade APACHE II. Estudo descritivo, realizado em uma Unidade de Terapia Intensiva de Trauma de um hospital de ensino. Foram utilizados os instrumentos Nursing Activities Score e APACHE II. A amostra foi composta por 32 pacientes, sendo a maioria do sexo masculino, adultos jovens, com politraumatismo, procedentes da Unidade de Emergência Referenciada, em tratamento cirúrgico, com alta da UTI. A média do Nursing Activities Score no período de internação foi de $72 \%$, e os dados mostraram uma correlação moderada entre a carga de trabalho e a gravidade do paciente, ou seja, quanto maior o escore, maior o risco de mortalidade apresentado pelos pacientes.

Descritores: Cuidados Críticos; Unidades de Terapia Intensiva; Carga de Trabalho; Cuidados de Enfermagem.

\section{ABSTRACT}

Severely injured patients with multiple and conflicting injuries present themselves to nursing professionals at critical care units faced with care management challenges. The goal of the present study is to evaluate nursing workload and verify the correlation between workload and the APACHE II severity index. It is a descriptive study, conducted in the Trauma Intensive Care Unit of a teaching hospital. We used the Nursing Activities Score and APACHE II as instruments. The sample comprised 32 patients, of which most were male, young adults, presenting polytrauma, coming from the Reference Emergency Unit, in surgical treatment, and discharged from the ICU. The average obtained on the Nursing Activities Score instrument was $72 \%$ during hospitalization periods. The data displayed moderate correlation between workload and patient severity. In other words, the higher the score, the higher the patient's mortality risk.

Descriptors: Critical Care; Intensive Care Units; Workload; Nursing Care.

\section{RESUMEN}

Los pacientes gravemente heridos, con lesiones múltiples y conflictivas se presentan ante los enfermeros de unidades de cuidados críticos como desafíos en gestión de atención. El estudio objetivó evaluar la carga de trabajo de enfermería y verificar la correlación entre la carga y el índice de gravedad APACHE II. Estudio descriptivo realizado en una Unidad de Terapia Intensiva de Trauma de un hospital de enseñanza. Fueron utilizados los instrumentos: Nursing Activities Score y APACHE II. Muestra compuesta por 32 pacientes, mayoría de sexo masculino, adultos jóvenes con politraumatismo, procedentes de la Unidad de Emergencia Referenciada, en tratamiento quirúrgico, con alta de la UTI. La media del Nursing Activities Score durante el período de internación fue de $72 \%$, y los datos mostraron una correlación moderada entre la carga de trabajo y la gravedad del paciente; es decir, ante mayor puntaje, mayor fue el riesgo de mortalidad presentado por los pacientes.

Descriptores: Cuidados Críticos; Unidades de Cuidados Intensivos; Carga de Trabajo; Atención de Enfermería. 


\section{INTRODUÇÃo}

O trauma decorrente de acidentes ou violência é considerado um problema de saúde pública e dependendo da sua gravidade pode resultar em incapacidades ou morte dos pacientes ${ }^{(1)}$. As lesões traumáticas variam em severidade, complexidade e nas circunstâncias como elas ocorrem ${ }^{(2)}$.

Em 2009, no Estado de São Paulo, os acidentes decorrentes de quedas corresponderam a $45 \%$ das internações hospitalares por causas externas, seguido por acidentes de transporte $(16,5 \%)$ e agressões $(3,8 \%)^{(3)}$. Segundo o Ministério da Saúde, dos 1.133.761 de óbitos ocorridos no Brasil em 2010, 141.771 foram decorrentes de causas externas. Neste mesmo ano, a taxa de óbito devido a essas mesmas causas foi de $9 \%$ no Estado de São Paulo e de $22 \%$ na cidade de Campinas $^{(4)}$.

O custo do atendimento às vítimas de causas externas para o sistema público de saúde em 2004 foi estimado em R\$2,2 bilhões, o das agressões de R\$119 milhões e dos acidentes de trânsito de R\$ 453 milhões, cujos gastos equivaleriam a cerca de $4 \%$ dos gastos totais com a saúde pública naquele ano ${ }^{(5)}$.

Os pacientes com trauma grave, que apresentam lesões múltiplas e conflitantes, apresentam-se aos enfermeiros de unidades de cuidados críticos como desafios na gestão da assistência. Os indivíduos, vítimas de trauma, usualmente apresentam risco de morte iminente e requerem assistência em unidades especializadas como é o caso das Unidades de Terapia Intensiva (UTI), cuja finalidade é proporcionar uma assistência de qualidade e melhorar o prognóstico dos pacientes.

A UTI caracteriza-se por ser uma área do setor hospitalar destinada ao cuidado contínuo aos pacientes em situações graves, os quais, além de necessitarem de recursos tecnológicos avançados, necessitam de uma equipe multiprofissional qualificada e especializada(6-7). Especificamente, para a gestão em enfermagem na UTI, existem legislações que orientam quanto ao número de profissionais necessários para atender esse perfil de pacientes e uma das recomendações é a avaliação dos pacientes por meio de um sistema de classificação de cuidados de enfermagem ${ }^{(6)}$.

Neste contexto, a utilização do Nursing Activities Score (NAS) tem sido destacada em estudos nacionais ${ }^{(8-}$ 10) e internacionais ${ }^{(11-12)}$ como uma ferramenta viável para a avaliação da carga de trabalho de enfermagem na UTI, com o objetivo de mensurar a porcentagem de tempo necessário para a realização das atividades de enfermagem requeridas pelo paciente durante as $24 \mathrm{~h}$ de internação ${ }^{(13)}$.

O NAS tem sido considerado um instrumento valioso para avaliar a carga de trabalho de enfermagem e estudos apontam associações entre a carga de trabalho e as variáveis: idade, sexo e gravidade dos pacientes ${ }^{(8,10)}$, infecção hospitalar ${ }^{(14)}$ e úlcera por pressão( ${ }^{(9)}$.

Em um estudo com o objetivo de identificar os fatores de risco para úlcera por pressão em pacientes críticos, verificou que o aumento do escore NAS resultou em um aumento no risco para desenvolver úlcera por

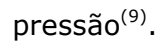

Outro aspecto importante a ser considerado na avaliação do paciente critico refere-se aos Índices de Gravidade, os quais quantificam, por meio de alterações clínicas e laboratoriais, o grau de disfunção orgânica de um paciente em estado grave. Esses índices auxiliam no processo de trabalho da UTI possibilitando 0 planejamento e a avaliação da assistência(15).

A correlação entre a carga de trabalho da enfermagem medida pelo NAS e os índices de gravidade, como o Acute Physiology and Chronic Health Evaluation (APACHE II), demonstram que quanto maior a gravidade do paciente, maior é a carga de trabalho da enfermagem ${ }^{(16)}$.

Considerando a inexistência de estudos que avaliem a carga de trabalho requerida pelos pacientes internados em uma UTI de trauma, o presente estudo teve como objetivos avaliar a carga de trabalho de enfermagem e verificar a correlação entre carga de trabalho e o índice de gravidade APACHE II.

\section{MÉTODO}

Trata-se de um estudo descritivo, realizado em uma UTI de Emergência Clínica e Trauma de um hospital de ensino do interior do Estado de São Paulo, caracterizado por ser uma instituição de atendimento terciário. A Unidade tem capacidade de atendimento para 20 leitos, sendo dez destinados à pacientes de emergência clínica e dez para vítimas de trauma, distribuídos em três postos e para o estudo foram considerados os pacientes internados no posto 201, com tempo de internação de pelo menos 24 horas, ambos os sexos e idade igual ou superior a 18 anos.

Para a coleta de dados utilizou-se o Nursing Activities Score (NAS), o índice de gravidade APACHE II e, uma ficha de caracterização de perfil clínico e demográfico dos pacientes.

O NAS tem como finalidade mensurar o tempo consumido pelas atividades de enfermagem na 
assistência ao paciente durante as 24 horas. É composto por 23 itens, divididos em sete categorias (atividades básicas, suporte ventilatório, cardiovascular, renal, neurológico, metabólico e intervenções específicas). A pontuação pode variar de zero a $177 \%$, podendo indicar a necessidade de mais de um profissional de enfermagem para o cuidado do paciente nas 24 $\operatorname{horas}^{(13,17)}$.

O APACHE II tem como objetivo avaliar o risco de óbito do paciente nas primeiras 24 horas de internação. Contém 12 variáveis, divididas em clínicas, fisiológicas e laboratoriais, como temperatura, pressão arterial média, frequência cardíaca, frequência respiratória, oxigenação por meio de valores gasométricos, pH arterial, sódio, potássio e creatinina séricas, hematócrito, glóbulos brancos e escala de coma de Glasgow. Cada variável recebe uma pontuação de zero a quatro, quanto maior o grau de desvio da normalidade apresentado, maior a pontuação. São atribuídos também, pontos à idade e a presença de doença crônica, a pontuação final varia de zero a 71 pontos $^{(18)}$.

A ficha de caracterização do perfil demográfico e clínico dos pacientes contém os seguintes dados dos pacientes: iniciais do nome, registro hospitalar, idade, sexo, diagnóstico médico, procedência, data de entrada e saída da UTI, tipo de tratamento e condições de saída da UTI.

O projeto de pesquisa obteve aprovação do Comitê de Ética em Pesquisa da Instituição (Processo: 00833614.4.0000.5404) com dispensa da assinatura do Termo de Consentimento Livre e Esclarecido (TCLE), pois os pacientes não foram submetidos a nenhuma intervenção durante a aplicação do instrumento.

Previamente à coleta, foi realizado um teste piloto com o NAS para familiarização com o instrumento e adequação de alguns aspectos do mesmo com o perfil dos pacientes da Unidade e de funcionamento desta.

Os dados foram coletados pela pesquisadora por meio dos registros médicos e de enfermagem contidos no prontuário do paciente e da passagem de plantão da equipe de enfermagem. A coleta de dados foi realizada por paciente, ou seja, um mesmo paciente foi acompanhado desde a sua admissão na UTI até a alta, transferência ou óbito.

Os dados coletados foram armazenados em uma planilha eletrônica do programa Microsoft Office Excel e analisados com auxílio de um profissional especializado. Para a análise dos dados foi utilizado o software estatístico SAS versão 9.2. Com o objetivo de avaliar a significância entre a média do NAS e as variáveis clínicas e demográficas dos pacientes, foram utilizados testes não paramétricos, como o teste de Mann-Whitney para as variáveis sexo, tipo de tratamento e condições de alta e, o teste de Kruskal-Wallis para diagnóstico médico e procedência.

Para avaliar a correlação entre a carga de trabalho resultante do NAS e o APACHE II, foi utilizado o coeficiente de correlação de Spearman. Os resultados foram considerados estatisticamente significantes se $p$ valor $<0,05$.

\section{RESULTADOS}

A amostra foi constituída por 32 pacientes, sendo $72 \%$ do sexo masculino e $28 \%$ do feminino, com idade média de 49,7 anos (DP +/- 22,44; Min=18; Max=86). As demais características dos pacientes estão apresentadas na Tabela 1.

O NAS foi aplicado no período de 73 dias consecutivos, totalizando 271 observações, cujos valores estão apresentados na Tabela 2.

Os itens do NAS que foram identificados com maior frequência estão apresentados na Tabela 3.

Foi possível também, comparar os escores do NAS com as variáveis demográficas e clínicas do estudo (Tabela 4).

Quanto à análise da correlação entre o NAS e o APACHE II, os resultados mostraram um coeficiente de Spearman de $0,45(p<0,001)$. 
Tabela 1: Distribuição do número, porcentagem, média e desvio padrão das variáveis demográficas e clínicas dos pacientes. Campinas, SP, Brasil, 2012.

\begin{tabular}{|c|c|c|c|c|c|}
\hline \multirow{2}{*}{ Variáveis } & \multicolumn{5}{|c|}{ Amostra } \\
\hline & $\mathbf{n}$ & $(\%)$ & Média (DP) & Mediana & Variação (Mín-Máx) \\
\hline \multicolumn{6}{|l|}{ Sexo } \\
\hline Masculino & 23 & $(72)$ & & & \\
\hline Feminino & 9 & $(28)$ & & & \\
\hline \multicolumn{6}{|l|}{ Idade } \\
\hline 18-31 anos & 6 & $(19)$ & & & \\
\hline $31-60$ anos & 15 & $(47)$ & & & \\
\hline$\geq 60$ anos & 11 & (34) & & & \\
\hline \multicolumn{6}{|l|}{ Procedência } \\
\hline Unidade de Emergência Referenciada & 15 & $(47)$ & & & \\
\hline Centro Cirúrgico & 12 & $(37,5)$ & & & \\
\hline Unidade de Internação & 5 & $(15,5)$ & & & \\
\hline \multicolumn{6}{|l|}{ Tipo de Tratamento } \\
\hline Cirúrgico & 27 & $(84)$ & & & \\
\hline Clínico & 5 & $(16)$ & & & \\
\hline Tempo de Permanência (dias) & & & $8,5(10,1)$ & 4,5 & $1-39$ \\
\hline \multicolumn{6}{|l|}{ Diagnóstico } \\
\hline Politrauma & 12 & $(37,5)$ & & & \\
\hline Fraturas & 7 & $(22)$ & & & \\
\hline Traumatismo intracraniano & 3 & $(9,5)$ & & & \\
\hline Outros* & 10 & $(31)$ & & & \\
\hline \multicolumn{6}{|l|}{ Condições de Alta } \\
\hline Sobreviventes & 28 & $(87,5)$ & & & \\
\hline Não sobreviventes & 4 & $(12,5)$ & & & \\
\hline APACHE II & & & $13,8(6,7)$ & 13 & $13-26$ \\
\hline
\end{tabular}
submandibular por angina de Ludwig e abdome agudo obstrutivo.

Tabela 2: Análise descritiva da carga de trabalho do Nursing Activities Score (NAS) nas primeiras 24 horas após admissão, no período de internação e na saída do paciente da Unidade. Campinas, SP, Brasil, 2012.

\begin{tabular}{cccccc}
\hline NAS & Média & DP & Mediana & Mínimo & Máximo \\
\hline Admissão & 85 & 12,28 & 83,7 & 65,1 \\
Período de internação & 72 & 12,76 & 68,5 & 5,7 \\
Alta & 77 & 11,31 & 76,6 & 103,7 & 58,2 \\
\hline
\end{tabular}

Tabela 3: Distribuição do número e porcentagem dos itens pontuados com maior frequência no Nursing Activities Score (NAS) durante as 271 observações realizadas. Campinas, SP, Brasil, 2012.

\begin{tabular}{|c|c|c|c|}
\hline Itens/subitens & Nursing Activities Score & $\mathbf{n}$ & $\%$ \\
\hline 2 & Investigações laboratoriais: bioquímicas e microbiológicas & 271 & 100 \\
\hline 3 & Medicação, exceto drogas vasoativas & 271 & 100 \\
\hline 17 & Medida quantitativa do débito urinário & 271 & 100 \\
\hline $7 a$ & $\begin{array}{l}\text { Suporte e cuidado aos familiares e pacientes que requerem dedicação exclusiva por cerca de } 1 \mathrm{~h} \text { em } \\
\text { algum plantão }\end{array}$ & 263 & 97 \\
\hline 11 & Tratamento para melhora da função pulmonar & 241 & 89 \\
\hline 9 & Suporte Respiratório & 240 & 88,5 \\
\hline $6 \mathrm{~b}$ & $\begin{array}{l}\text { Realização do(s) procedimento(s) mais do que } 3 \text { vezes em } 24 \mathrm{~h} \text { ou com } 2 \text { enfermeiros em qualquer } \\
\text { frequência }\end{array}$ & 233 & 86 \\
\hline 5 & Cuidados com drenos - Todos (exceto sonda gástrica) & 212 & 78 \\
\hline $8 a$ & $\begin{array}{c}\text { Realização de tarefas de rotina tais como: processamento de dados, solicitação de exames, troca de } \\
\text { informações profissionais }\end{array}$ & 210 & 77,5 \\
\hline 10 & Cuidado com vias aéreas artificiais. Tubo endotraqueal ou cânula de traqueostomia & 186 & 69 \\
\hline
\end{tabular}

Tabela 4: Comparação das variáveis do estudo (sexo, diagnóstico, procedência, tipo de tratamento e saída da unidade) com a média

\begin{tabular}{|c|c|c|c|c|c|c|c|}
\hline Variável & $\mathbf{N}$ & Média & Desvio-padrão & Mínimo & Mediana & Máximo & p-valor \\
\hline Sexo & & & & & & & $0,403^{*}$ \\
\hline Masculino & 21 & 70,6 & 12,6 & 55,3 & 67,9 & 103,8 & \\
\hline Feminino & 7 & 75,6 & 13,6 & 56,5 & 77,9 & 94,6 & \\
\hline Diagnóstico & & & & & & & $0,054 * *$ \\
\hline Traumatismo intracraniano & 3 & 67,9 & 3,8 & 64,6 & 67,1 & 72 & \\
\hline Politrauma & 12 & 70,1 & 12,8 & 55,3 & 68,1 & 103,8 & \\
\hline Fratura & 6 & 64,7 & 9,5 & 56,4 & 60,5 & 81,4 & \\
\hline Outros*** & 7 & 82,5 & 12,6 & 61 & 84,5 & 97,9 & \\
\hline Procedência & & & & & & & $0,636 * *$ \\
\hline Centro Cirúrgico & 11 & 71,8 & 13,9 & 56,5 & 68,2 & 103,8 & \\
\hline UER & 13 & 70,7 & 12 & 55,3 & 67,9 & 97,9 & \\
\hline Unidade de Internação & 4 & 75,3 & 11,3 & 61 & 76,7 & 86,7 & \\
\hline Tratamento & & & & & & & $0,047 *$ \\
\hline Cirúrgico & 25 & 70,1 & 12,2 & 55,3 & 67,9 & 103,8 & \\
\hline Clínico & 3 & 86,4 & 8,4 & 77,9 & 86,7 & 94,6 & \\
\hline Condições de alta & & & & & & & $0,170 *$ \\
\hline Sobrevivente & 24 & 70,4 & 12,5 & 55,3 & 68,1 & 103,8 & \\
\hline Não Sobrevivente & 4 & 80 & 12,8 & 66 & 79,3 & 94,6 & \\
\hline
\end{tabular}

* p-valor obtido por meio do teste de Mann-Whitney;

** p-valor obtido por meio do teste de Kruskal-Wallis

*** Insuficiência respiratória aguda, choque cardiogênico, septicemia, adenocarcinoma de colon, acidente vascular encefálico hemorrágico, abscesso submandibular por angina de Ludwig e abdome agudo obstrutivo. 


\section{DISCUSSÃO}

Os pacientes foram na maioria do sexo masculino, adultos jovens, procedentes do Centro Cirúrgico e da Unidade de Emergência Referenciada, com os diagnósticos de politrauma e fratura, e em tratamento cirúrgico. Esses achados se assemelham aos estudos que caracterizaram os pacientes atendidos no Pronto Socorro de um hospital público de referência em trauma no sul do país ${ }^{(19)}$ e aos dados do Ministério da Saúde, os quais destacam que $68,7 \%$ das internações hospitalares ocorridas por causas externas no Estado de São Paulo em 2009 foram em indivíduos do sexo masculino, 50\% entre indivíduos de 20-49 anos e 45\% decorrente de quedas, seguido de $16,5 \%$ por acidentes de transporte ${ }^{(3)}$.

Em relação ao tempo de internação, este foi superior a outros estudos realizados em UTI Adulto e de Cardiologia(20-21), e inferior ao estudo em UTI Geral de Adultos $^{(9,14)}$. No entanto, ressalta-se que não há estudos disponíveis com este mesmo perfil de pacientes para a realização de comparações.

A carga de trabalho de enfermagem foi superior quando comparado com estudos realizados em UTI Adulto de dois hospitais de ensino do Estado de São Paulo ${ }^{(9-21)}$ como também em um estudo realizado na Espanha ${ }^{(12)}$. Em contrapartida, a média obtida do NAS no presente estudo está próxima de uma UTI Cardiológica $^{(22)}$ e inferior a um estudo realizado na Noruega ${ }^{(11)}$.

Obteve-se uma diminuição da média do NAS em relação ao NAS de admissão e alta. No entanto, esses pacientes são transferidos para as unidades de internação com uma carga considerada elevada, e uma das justificativas deve-se ao fato da instituição não dispor de uma unidade de cuidados semi-intensivos para receber esses pacientes e por ser uma instituição de atendimento terciário. A diminuição da média do NAS na alta também foi observada em outro estudo, o qual mostrou que a média do NAS na alta é maior em pacientes idosos do que em adultos ${ }^{(10)}$.

Ao se comparar a carga de trabalho medida pelo NAS com as variáveis demográficas e clínicas do estudo, observa-se uma média do NAS maior no sexo feminino, nos pacientes com diagnóstico clínico, nos que tiveram como procedência as Unidades de Internação e os que evoluíram a óbito. Embora não tenha resultado em diferenças estatísticas, verifica-se que os pacientes em tratamento clínico obtiveram maiores médias do NAS quando comparados com os pacientes que receberam tratamento cirúrgico.
Os itens e subitens do NAS observados com maior frequência corroboram com dados encontrados em outro estudo realizado em UTI de Adulto $^{(21)}$, os quais são identificados como atividades de rotina de uma UTI.

A correlação significativa entre a gravidade dos pacientes e a carga de trabalho de enfermagem requerida pelos mesmos foi de 0,45 mostrando uma correlação positiva, mas de moderada magnitude, entre essas duas variáveis. No entanto, outro estudo realizado em uma UTI Geral de Adultos, constatou uma correlação de forte magnitude entre esses dois índices $(r=0,82)^{(16)}$. o que pode ser justificado pelo perfil desses pacientes.

Destaca-se a ausência de estudos com o mesmo perfil de pacientes, não sendo possível comparar os resultados. Além disso, devido ao tamanho da amostra e pela coleta ter sido realizada em uma única UTI no Brasil, especializada em atendimento ao trauma, não é possível fazer generalização dos resultados.

\section{CONCLUSÃO}

Os dados obtidos neste estudo permitiram caracterizar os pacientes internados na referida Unidade, sendo em sua maioria pacientes adultos jovens, do sexo masculino, politraumatizados e, em tratamento cirúrgico.

Destaca-se a importância da utilização do NAS para avaliar a carga de trabalho requerida em uma UTI de atendimento ao paciente com trauma. A carga de trabalho requerida pelos pacientes foi elevada na admissão, período de internação e alta e não diferiu entre as variáveis demográficas e clínicas dos pacientes. Os itens do NAS que apareceram em maior frequência estão relacionados às atividades de rotina da UTI.

Obteve-se uma correlação moderada entre a carga de trabalho e o APACHE II, ou seja, quanto maior o NAS, maior o risco de mortalidade dos pacientes.

Os resultados mostraram que os pacientes assistidos em uma UTI especializada em trauma, apresentam elevadas cargas de trabalho e isto pode repercutir diretamente na qualidade e custo da assistência. Tratase do primeiro estudo que avaliou a carga de trabalho requerida pelos pacientes em uma UTI especializada em atendimento à vitima de trauma e, outros estudos serão necessários para avaliar associações entre carga de trabalho e índices de gravidade específicos para esse perfil de clientela. 


\section{REFERÊNCIAS}

1. Mathers CD; Boerma T; Fat DM. Global and regional causes of death. British Medical Bulletin. 2009; 92:7-32.

2. Crossan L; Cole E. Nursing challenges with a severely injured patient in critical care. Nursing in Critical Care. 2013; 18(5):236244.

3. DATASUS [Internet]. Brasília: Ministério da Saúde (BR) [cited 2011 dec 15]. Proporção de internações hospitalares (SUS) por causas externas, 2009. Available from:

http://tabnet.datasus.gov.br/cgi/tabcgi.exe?idb2010/d14.def.

4. DATASUS [Internet]. Brasília: Ministério da Saúde (BR) [cited 2011 dec 15]. Estatísticas de mortalidade. Óbitos por residência segundo município, 2010 Available from:

http://tabnet.datasus.gov.br/cgi/tabcgi.exe?sim/cnv/pext10sp.de f.

5. RI, Cerqueira DRC, Lobão WJA, Carvalho AXY. Os custos da violência para o sistema público de saúde no Brasil: informações disponíveis e possibilidades de estimação. Cad. Saúde Pública. 2009;25(1):29-36.

6. Resolução RDC $N^{\circ} 7$, de 24 de fevereiro de 2010. Dispõe sobre os requisitos mínimos para funcionamento de unidades de terapia intensiva e dá outras providências. [cited 2012] Available from: http://www.saude.mg.gov.br/atos_normativos/legislacaosanitaria/estabelecimentos-de-saude/uti/RDC7_ANVISA\%20240210.pdf.

7. Chaves LDP, Laus AM, Camelo SH. Ações gerenciais e assistenciais do enfermeiro em unidade de terapia intensiva Rev. Eletr. Enf. [Internet]. 2012;14(3):671-8. Available from: http://www.fen.ufg.br/revista/v14/n3/v14n3a25.htm. 8. Padilha KG, Sousa RMC, Garcia PC, Bento ST, Finardi EV, Hatarashi RHK. Nursing workload and staff allocation in an intensive care unit: a pilot study according to Nursing Activities Score (NAS). Intensive Crit. Care Nurs. 2010;26:108-13. 9. Cremasco MF, Wenzel F, Sardinha FM, Zanei SSV, Whitaker IY. Úlcera por pressão: risco e gravidade do paciente e carga de trabalho de enfermagem. Acta paul. enferm. 2009;22(spe):897902.

10. Sousa RMC, Padilha KG, Nogueira LS, Miyadahira AMK, Oliveira VCR. Carga de trabalho de enfermagem requerida por adultos, idosos e muito idosos em Unidade de Terapia Intensiva. Rev. Esc. Enferm. USP. 2009; 43(spe2):1284-1291.

11. Stafseth SK, Solms D, Bredal IS. The characterisation of workloads and nursing staff allocation in intensive care units: a descriptive study using the Nursing Activities Score for the first time in Norway. Intensive Crit. Care Nurs. 2011;27(5):290-94. 12. Adell AB, Campos RA, Rey MC, Bellmunt JQ, Rochera ES, Muñoz JS, Canuto MS, López RT, Bou MY. Nursing Activity Score (NAS). Nuestra experiencia con un sistema de cómputo de cargas de enfermería basado en tiempos. Enferm Intensiva.

2005;16(4):164-73.

13. Miranda DR, Nap R, Rijk A, Schaufeli W, Iapichino G. et al. Nursing Activities Score. Crit Care Med. 2003;31(2):374-82.

14. Cyrino CMA, Dell'Acqua MCQ. Sítios assistenciais em Unidade de Terapia Intensiva e relação do nursing activities score com a infecção hospitalar. Esc. Anna Nery. 2012; 16(4): 712-718. 15. Andrei AM, Moura Junior DF, Almeida FP, Lagudis S. Índices prognósticos em terapia intensiva. In: Knobel E. Condutas no paciente grave. $3^{a}$ ed. São Paulo: Atheneu; 2006. p. 2055-79. 16. Nogueira LS, Santos MR, Mataloun SE, Moock M. Nursing Activities Score: comparação com o índice APACHE II e a mortalidade em pacientes admitidos em unidade de terapia intensiva. Rev Brasileira de Terapia Intensiva 2007;19(3):32730.

17. Queijo AF, Padilha KG. Nursing Activities Score (NAS): crosscultural adaptation and validation to portuguese language. Rev Esc Enferm USP 2009; 43(Esp):1018-25.

18. Knaus WA, Draper EA, Wagner DP, Zimmerman JE. APACHE II: a severity of disease classification system. Crit Care Med. 1985;13(10):818-29.

19. Di Credo PF, Felix JVC. Perfil dos pacientes atendidos em um hospital de referência ao trauma em Curitiba: implicações para a enfermagem. Cogitare Enferm. 2012;17(1):126-31.

19. Coelho FUA, Queijo AF, Andolhe R, Gonçalves LA, Padilha KG. Carga de trabalho de enfermagem em unidade de terapia intensiva de cardiologia e fatores clínicos associados. Texto Contexto Enferm. 2011;20(4):735-41.

21. Panunto MR, Guirardello EB. Nursing workload in an intensive care unit of a teaching hospital. Acta Paul Enferm.

2012;25(1):96-101.

22. Ducci AJ. Padilha KG. Nursing activities score: a comparative study about retrospective and prospective applications in intensive care units Acta Paul Enferm. 2008;21(4):581-7.

Artigo recebido em 04/03/2013.

Aprovado para publicação em 28/02/2014.

Artigo publicado em 30/06/2014. 